\title{
Compact hard X-ray grating interferometry for table top phase contrast micro CT
}

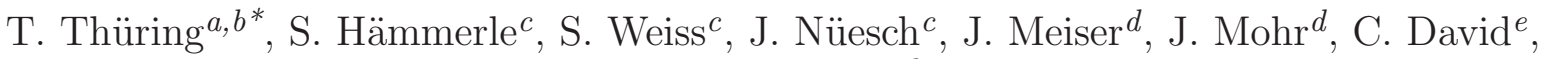 \\ M. Stampanoni ${ }^{a, b}$ \\ ${ }^{a}$ Swiss Light Source, Paul Scherrer Institute, Villigen PSI, Switzerland \\ ${ }^{b}$ Institute for Biomedical Engineering, Swiss Federal Institute of Technology, Zurich, \\ Switzerland \\ ${ }^{c}$ SCANCO Medical AG, Brüttisellen, Switzerland \\ ${ }^{d}$ Karlsruhe Nano Micro Facility (KNMF), Karlsruhe Institute of Technology (KIT), Karlsruhe, \\ Germany \\ ${ }^{e}$ Laboratory for Micro- and Nanotechnology, Paul Scherrer Institute, Villigen PSI, Switzerland
}

\begin{abstract}
Today's commercial X-ray micro computed tomography (CT) specimen systems are based on microfocus sources, 2D pixel array cameras and short source-to-detector distances (i.e. cone-beam configurations). High resolution is achieved by means of geometric magnification. The further development of such devices to acquire phase and scattering contrast images can dramatically enhance their range of applications. Due to the compact geometries, which imply a highly diverging beam, the gratings must be curved to maintain highest imaging performance over a large field of view. We report about the implementation of extremely compact Talbot and TalbotLau type grating interferometers which are compatible to the geometry of typical micro CT systems. For the analytical description of the imaging system, formulas are presented describing the dependency of the sensitivity on geometric parameters, camera and source parameters. Further, the imaging pipeline consisting of the data acquisition protocol, radiographic phase retrieval and tomographic image reconstruction is illustrated. The reported methods open the way for an immediate integration of phase and scattering contrast imaging on table top X-ray micro CT scanners.
\end{abstract}

Keywords: Phase contrast imaging, grating interferometry, compact setup, curved gratings, phase contrast micro CT

\section{INTRODUCTION}

Among the variety of available phase contrast techniques, X-ray grating interferometry (GI) has favorable properties for the application on conventional X-ray tubes, ${ }^{1,2}$ which offers a huge potential for the implementation in commercial systems. Exploiting differential phase contrast (DPC) and also dark field contrast (DC) ${ }^{3}$ imaging can yield a significant enhancement of the contrast-to-noise ratio compared to conventional, absorption based X-ray imaging. ${ }^{4-6}$

A promising industrial application of GI are micro CT scanners, which are usually standalone or table top systems and provide high resolution 3D imaging functionality for various kinds of specimen. High resolution is achieved by high brilliance microfocus X-ray tubes, area detectors with small pixels and by geometric magnification. Promising is the fact that the X-ray energy and the spatial resolution are in a range where current GI technology is expected to yield highest performance. ${ }^{7}$ In addition, GI requires a certain propagation distance between sample and detector, giving a natural consistency with the principle of geometric magnification.

Up to now, the application of GI on micro CT systems has been limited due to their typically compact geometry. A short source-to-detector distance and a large area pixel detector imply a high beam divergence, leading to a curved wave front, or a fan-beam. The limitation is then given by the incompatibility of the curved

*E-mail: thomas.thuering@psi.ch

Medical Imaging 2013: Physics of Medical Imaging, edited by Robert M. Nishikawa, Bruce R. Whiting, Christoph Hoeschen, Proc. of SPIE Vol. 8668, 866813 · (c) 2013 SPIE · CCC code: 1605-7422/13/\$18 · doi: 10.1117/12.2006865 
wave front and the planar gratings of an interferometer. The consequence is a position dependent sensitivity, which has its maximum at the optical axis and decays rapidly when moving outwards. In other words, using planar gratings in a compact fan-beam system leads to a severe reduction of the field of view (FOV). Since this effect is mainly related to the high aspect ratios of absorption gratings, this limitation becomes even worse at higher energies.

We have developed GI for compact micro CT systems, where the FOV is not limited by the gratings and the photon energy. The development involved a novel grating fabrication method, aiming to obtain curved gratings. Such gratings allow matching the grating shape to the divergent beam, which solves the above mentioned limitations. The technical realization was solved by modifying the fabrication process to work with a more flexible substrate than the conventionally used silicon.

Up to now, the application of curved gratings has already been successfully demonstrated for a compact Talbot interferometer implemented on a setup with a microfocus source. ${ }^{8}$ Here, we report on the application of GI with bendable gratings by using two different designs which are compatible to commercial micro CT scanners. We further report on the sensitivity of these designs and on the imaging pipeline for micro CT applications consisting of data acquisition, radiographic phase retrieval and 3D image reconstruction.

\section{THEORY AND METHODS}

Figure 1 shows a schematic of a compact grating interferometer with curved gratings. The gratings are labeled by G0 (source grating), G1 (beam splitter grating) and G2 (analyzer grating) and their pitches by $p_{0}, p_{1}$ and $p_{2}$. The radius of curvature always corresponds to the distance from the focal spot of the source to the grating. G1 generates a periodic interference pattern (fringes), which is transversely shifted if an object in front of G1 refracts the beam. G2 is used to analyze the fringes and to detect this transversal shift, from which the angle of refraction can be calculated in every pixel. ${ }^{9}$

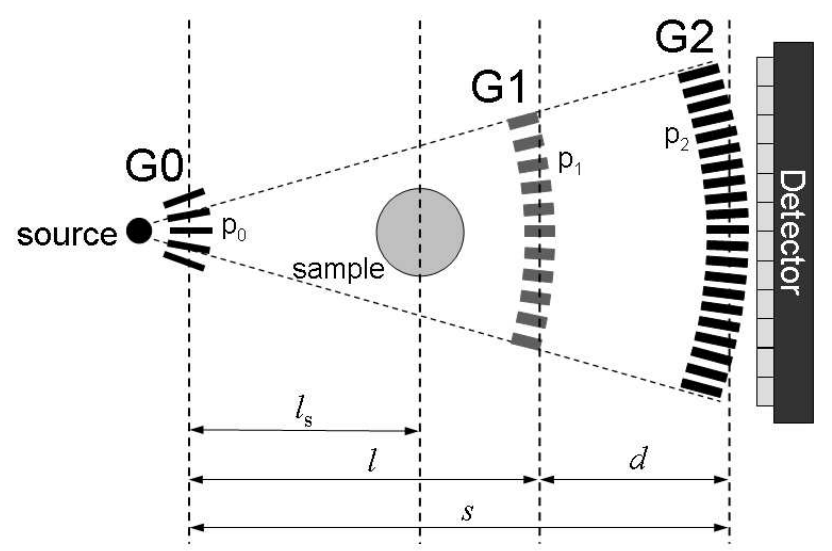

Figure 1. Schematic of a compact grating interferometer in a fan-beam geometry. Gratings are curved due to the high beam divergence and the high grating aspect ratios.

\subsection{Grating design}

State-of-the-art gratings based on planar wafers encounter a severe limitation when applied to a highly divergent beam in a compact fan-beam (or cone-beam) imaging system. Due to the typically high aspect ratio of the grating structures, a significant change of the grating transmission function occurs for X-rays at high incident angles. This consequently reduces the visibility of the interference fringes measured at these angles and degrades the detectability of the DPC signal. Essentially, this implies a severe reduction of the FOV. ${ }^{8}$

We have developed new gratings by using a modified LIGA process, ${ }^{10}$ aiming at fabricating the gratings on a more flexible substrate. Since the conventionally used silicon wafers (thicknesses of $200-500 \mu \mathrm{m}$ ) tend to be brittle and can only be curved down to a radius of a few tens of centimeters, ${ }^{11}$ the process was adapted for 
manufacturing on titanium substrates. Due to the low substrate thickness of only $50 \mu \mathrm{m}$, it offers high flexibility and allows a radius of curvature of a few centimeters without damaging the grating. In particular for a TalbotLau setup, the radius of curvature of the source grating, G0, needs to be small in order to maintain a compact geometry.

In summary, grating interferometry with curved gratings solves the problem of the incompatibility between the divergent wave front and planar, high aspect ratio gratings and opens the path to phase contrast and dark field imaging on commercial micro CT scanners.

\subsection{Sensitivity}

Despite of the constraint of having a compact geometry, there are still many degrees of freedom for the interferometer design. Therefore, maximizing the sensitivity, or the minimum detectable refractive angle, ${ }^{12}$ of the differential phase (or refractive angle) measurement is essential to achieve highest performance. For the Talbot and the Talbot-Lau interferometer, the sensitivity is given by ${ }^{13}$

$$
\alpha_{\min }=\frac{p_{2}}{2 \pi d} \frac{\sqrt{2 \chi_{\mathrm{d}}}}{V \sqrt{N}}
$$

where $V$ is the fringe visibility, $N$ the detector counts and $\chi_{\mathrm{d}}$ is a detector specific parameter, relating the output counts $(N)$ to the noise variance by $\sigma_{\mathrm{d}}^{2}=\chi_{\mathrm{d}} N .^{14}$ If the DPC projections are in addition flat field corrected, Eq. (1) has to be multiplied by the factor $\sqrt{2}$.

Eq. (1) can be used for the optimization of the interferometer geometry. For the herein presented experiments, the sensitivity of a Talbot and a Talbot-Lau setup has been experimentally analyzed to verify Eq. (1). A Talbot interferometer with $p_{1}=4.12 \mu \mathrm{m}, p_{2}=2.4 \mu \mathrm{m}$ and $s=326 \mathrm{~mm}$ and a symmetric Talbot-Lau interferometer with $p_{0}=p_{1}=p_{2}=2.4 \mu \mathrm{m}$ and $s=200 \mathrm{~mm}$ have been set up. Both are designed for a mean X-ray energy of $28 \mathrm{keV}$. The absorption grating material is gold and the phase grating material is Nickel, generating a periodic phase shift of $\pi$.

\subsection{Resolution}

The minimum achievable effective pixel size of a system is limited by the focal spot of the source, $w$, and the pixel size of the detector, $p_{\mathrm{d}}$, and is given by ${ }^{8}$

$$
p_{\text {eff }}=\frac{w \cdot p_{\mathrm{d}}}{w+p_{\mathrm{d}}}
$$

The corresponding geometric magnification is

$$
M_{\mathrm{s}}=\frac{p_{\mathrm{d}}+w}{w} .
$$

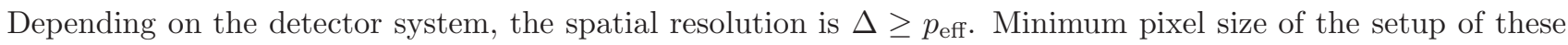
experiments is $p_{\mathrm{d}}=24 \mu \mathrm{m}$. With a spot size range of 5 to $10 \mu \mathrm{m}$, the minimum achievable effective pixel size is 4.1 to $7.1 \mu \mathrm{m}$, which is in the resolution range of a typical micro CT system.

\subsection{Imaging pipeline}

Images are acquired by using the standard phase stepping approach. ${ }^{9}$ Even though the gratings are curved, phase stepping is not performed on an arc trajectory but on a straight line. The error in the phase stepping distance at higher fan angles caused by this approximation is negligible and does not reduce imaging performance.

In $2 \mathrm{D}$ radiography, the phase stepping data is processed by using a Fourier approach, ${ }^{15}$ where the output is the DPC image. For the retrieval of the line integral, or the projected phase, an integration of the DPC image must be performed. However, even for images with a moderate SNR, this integration often leads to severe stripe artifacts, destroying details in the image and impeding the subsequent analysis of the radiographs. In order to suppress such artifacts, an iterative method has been developed which is based on a regularization approach. ${ }^{16}$ Fig. 2 shows a DPC radiography of a wasp, the direct integration, the regularized integration and the vertical 


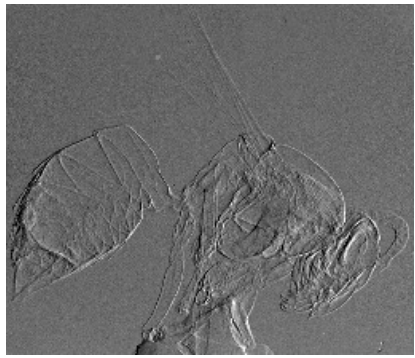

(a)

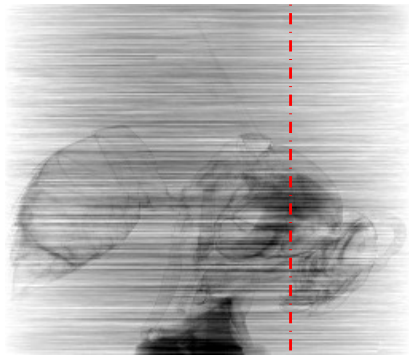

(b)

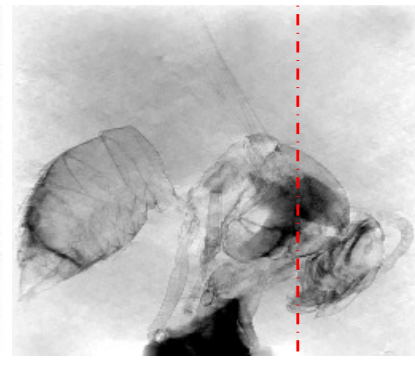

(c)

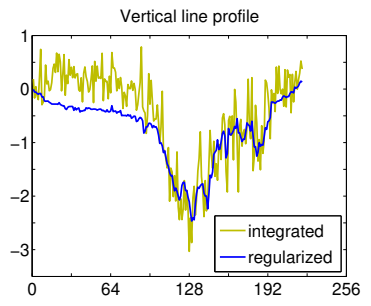

(d)

Figure 2. Phase radiography of a wasp. a) raw DPC image, b) direct integration, c) regularized integration, d) vertical line profiles through b) and c) at the dashed line. In c), the horizontal stripe artifacts could be removed and resolution was maintained.

line profiles through the images. The regularized image and the line profiles clearly indicate the benefits of the iterative method, yielding in a more robust phase retrieval.

In tomography mode (3D), DPC projections of the object as well as flat images (without object) are recorded using a 360 degrees scan. In the ideal case, the flat fields used to correct the projections are acquired at the beginning. However, due to the typically small periodicity of the gratings, the grating interferometer is highly sensitive to mechanical drift of any of the gratings or of the focal spot of the source. If drift becomes an issue, it causes changes in the reference phase values and the flat field correction fails. Since the sensitivity to drift is higher for smaller grating pitches, it is likely to occur for compact geometries. If drift is small enough (i.e. at least below one period of the drifting grating), a first order phase map (offset and gradient) can be calculated and subtracted from the processed DPC projections. This is based on the assumption that a small linear drift of one grating relative to the other causes a constant offset in the DPC image, whereas a slight relative rotation causes a gradient. Offset and gradient can be calculated by fitting a linear 2D function to the background pixels (no object) of the DPC projection image, which makes use of the a-priori knowledge that the phase has to be zero at these pixels.

If drift becomes too severe (larger than a period of the drifting grating), the assumptions for the first order corrections are invalid, the flat field correction fails and image artifacts will appear. In this case, acquiring flat field images between the object projections of the tomographic scan is a robust method. In practice, one or multiple flat field images are acquired before and after a fixed number of object projections. Projections which have been taken in between two flat fields can then be corrected by using an interpolated phase map calculated from these flat fields.

For the reconstruction of the DPC projections into a 3D volume, the beam divergence, implying a cone-beam geometry, has to be taken into account. A standard method for the reconstruction of cone-beam CT data is the Feldkamp-David-Kress (FDK) algorithm. ${ }^{17}$ It can, analog to the parallel beam geometry, easily be modified to handle differential phase data by using the appropriate filter. ${ }^{18}$

\section{RESULTS}

\subsection{Sensitivity measurements}

The purpose of the sensitivity measurements was to verify Eq. (1) and to compare the compact Talbot and Talbot-Lau interferometer, respectively. Micro CT systems usually operate with a beam of high spatial coherence, and thus, both types are feasible configurations. Since the optimization of the two interferometer types works differently, it is difficult to establish identical conditions for a comparison. Here, Talbot and Talbot-Lau interferometer have been individually optimized using identical hardware for source and detector, with the aim of getting an idea of which configuration has superior properties for micro CT systems. The X-ray source is a Hamamatsu L10101 microfocus tube with a minimum focal spot size of $5 \mu \mathrm{m}$. The detector is a Princeton Instruments CCD camera with $2084 \times 2084$ pixels and a pixel size of $24 \mu \mathrm{m}$. X-rays are converted to visible light by a $\mathrm{Gd}_{2} \mathrm{O}_{2} \mathrm{~S}: \mathrm{Tb}$ screen. 
Sensitivity measurements were performed at different voltage values of the X-ray source (40 to $60 \mathrm{kV}$ ). Since the Talbot interferometer relies on spatially coherent X-rays, the power of the X-ray tube was set to the minimum $(4 \mathrm{~W})$, such that the focal spot is small $(w \approx 5 \mu \mathrm{m})$. On the other hand, for the Talbot-Lau interferometer, the tube current was set to the maximum $(I=200 \mu \mathrm{A})$ to maximize the beam intensity. The expected focal spot size of the source for this setting is $w \approx 10 \mu \mathrm{m}$.

100 phase stepping scans by using 16 steps and $8 \mathrm{~s}$ of exposure time per step were acquired. Each time before and after ten phase stepping scans, a flat field scan has been taken, resulting in a total of 11 flat fields. The phase stepping scans have then been corrected with the flat fields by using phase map interpolation (see above). From the resulting DPC projections, the standard deviation of the differential phase value, $\sigma_{\varphi}$, was calculated over a small region of interest. The sensitivity was then calculated by using $\alpha_{\min }=\frac{p_{2}}{2 \pi d} \sigma_{\varphi}$.

Figure 3 shows the visibility and the sensitivity as a function of tube voltage for the Talbot and the TalbotLau interferometer. In addition to the sensitivity measurements (plotted with solid lines), the sensitivity was evaluated by using Eq. (1) (dashed lines). The noise parameter of the detector was measured as $\chi_{\mathrm{d}, \mathrm{T}}=7.1$ and $\chi_{\mathrm{d}, \mathrm{TL}}=8.9$ for the Talbot $(\mathrm{T})$ and the Talbot-Lau (TL) interferometer, respectively, and the visibility $V$ was taken from Figure 3a (measured). Both interferometers show a different behavior when changing the voltage, which is mainly related to the different acceptances of the spectral bandwidth. While the Talbot interferometer accepts a bandwidth from roughly 15 to $80 \mathrm{keV}$, the Talbot-Lau type accepts energies only between 21 and $42 \mathrm{keV}$. This is mainly due to the different Talbot orders of the two systems, which are 0.83 and 3 for the Talbot and the Talbot-Lau type, respectively. According to Figure 3b, the Talbot-Lau interferometer performs clearly better than the Talbot interferometer in the given voltage range and for otherwise identical scan parameters. It is important to emphasize that the sensitivity is exposure time dependent. A longer exposure time would further decrease $\alpha_{\min }$ proportional to $1 / \sqrt{t}{ }^{13}$

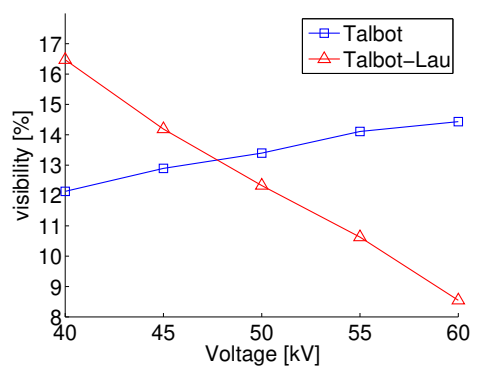

(a)

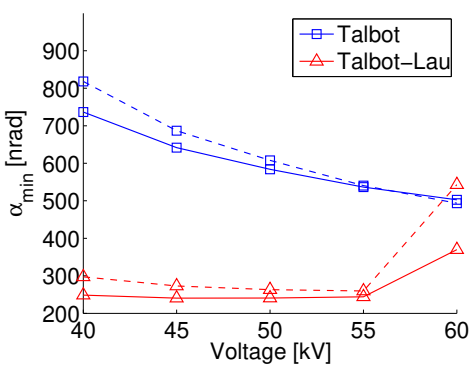

(b)

Figure 3. a) Visibility and b) sensitivity evaluation of the Talbot and the Talbot-Lau interferometer. For the Talbot interferometer, $\alpha_{\min }$ decreases for higher voltages, because both, the visibility $V$ and the beam intensity $I_{0}$, increase. The optimum is expected to be at even higher voltages. For the Talbot-Lau interferometer, the sensitivity seems to be almost independent on the tube voltage. Any increase of the voltage reduces the visibility but increases the beam intensity. Still, an optimum is observed at $45 \mathrm{kV}$. The dashed lines in b) show the analytical sensitivity as obtained from Eq. (1).

\subsection{Imaging}

Figure 4 shows a tomographic scan of a bacon fixed in a falcon tube by using the Talbot-Lau type interferometer. The tube voltage was $40 \mathrm{kV}$ and the current was $200 \mu \mathrm{A}$. The mean visibility across the FOV was $V=16.6 \%$. 8 phase steps were acquired with an exposure time of $10 \mathrm{~s}$ per step. Using 2000 projections, the total acquisition time for the sample was approx. 44 hours. After every ten projections, 4 flat fields have been acquired, resulting in a total of 800 flat fields and an acquisition time of 22 hours. Reconstruction has been performed by using an FDK-based cone-beam algorithm.

Comparing absorption and phase image, sufficient contrast for distinguishing fatty tissue from muscle is provided by both contrast modes. However, the contrast-to-noise ratio (CNR) is clearly superior in the phase image. High resolution details vanish in the absorption image, but remain visible in the phase image due to the enhanced CNR. 


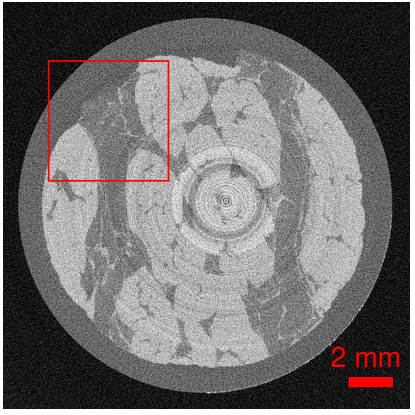

(a)

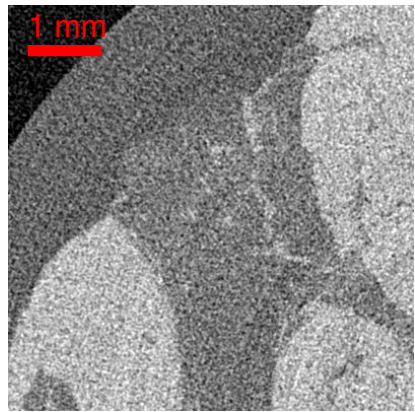

(b)

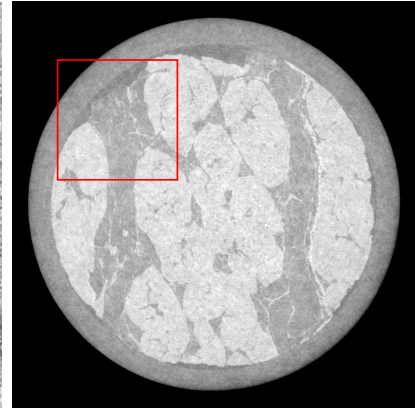

(c)

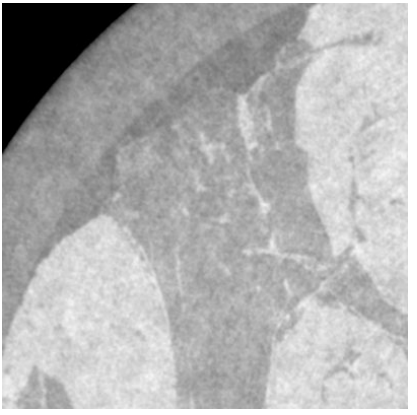

(d)

Figure 4. Tomographic slice of a bacon using the Talbot-Lau type interferometer with an effective pixel size of $p_{\text {eff }}=13 \mu \mathrm{m}$, $V=45 \mathrm{kV}$ and $I=200 \mu \mathrm{A}$. a) Absorption image and b) ROI inside rectangle, c) phase image and d) ROI inside rectangle. While both, absorption and phase, provide sufficient contrast to distinguish fat tissue from muscle tissue, the contrastto-noise properties, or the sensitivity, is clearly superior in phase. High resolution details vanish in the absorption image but can be resolved in phase.

\section{CONCLUSIONS}

Curved gratings are the key components for a successful transfer of the grating interferometer technology into commercial micro CT scanners. Matching the grating curvature to the divergent beam solves the problem of a reduced FOV, which occurs when using planar state-of-the-art gratings. Especially for compact Talbot-Lau interferometers, the development of a new grating substrate was unavoidable in order to achieve an extremely small radius of curvature for the source grating (G0).

Regularized integration for retrieving the projected phase from the DPC image represents a powerful tool for artifact-free phase contrast radiography. For 3D imaging, a standard FDK based algorithm with a modified filter can be used to reconstruct the cone-beam DPC projections into a 3D volume.

Interferometer drift represents a problem for GI on micro CT systems. Current solutions to drift are first order phase map corrections and phase map interpolation, which mainly affect the scan time and not the dose to the sample.

A Talbot- and a Talbot-Lau type interferometer with curved gratings, which are both compatible with typical micro CT geometries, have been optimized independently and compared by investigating the sensitivity. Despite of the microfocus source, which provides high spatial coherence for a two gratings based Talbot interferometer, the Talbot-Lau interferometer accomplishes a higher sensitivity for otherwise identical scan parameters. On the other hand, the Talbot type interferometer has several advantages over the Talbot-Lau type for the application of micro CT systems, as for instance less beam attenuation, a simpler alignment, more space for sample movements due to the asymmetric geometry or a higher range of geometrical magnifications. With further improvement of the X-ray source brilliance in the future, the Talbot interferometer will certainly comply with the demands for highly sensitive phase contrast and scattering imaging on micro CTs.

This work was partly carried out with the support of the Karlsruhe Nano Micro Facility (KNMF, www.kit.edu/knmf), a Helmholtz Research Infrastructure at Karlsruhe Institute of Technology (KIT, www.kit.edu).

\section{REFERENCES}

[1] Pfeiffer, F., Weitkamp, T., Bunk, O., and David, C., "Phase retrieval and differential phase-contrast imaging with low-brilliance X-ray sources," Nature Physics 2, 258-261 (2006).

[2] Engelhardt, M., Baumann, J., Schuster, M., Kottler, C., Pfeiffer, F., Bunk, O., and David, C., "Highresolution differential phase contrast imaging using a magnifying projection geometry with a microfocus x-ray source," Applied Physics Letters 90(22), 224101 (2007).

[3] Pfeiffer, F., Bech, M., Bunk, O., Kraft, P., Eikenberry, E., Brönnimann, C., Grünzweig, C., and David, C., "Hard-X-ray dark-field imaging using a grating interferometer," Nature Materials 7(2), 134-137 (2008). 
[4] Momose, A. and Fukuda, J., "Phase-contrast radiographs of nonstained rat cerebellar specimen," Medical Physics 22(4), 375-379 (1995).

[5] McDonald, S. A., Marone, F., Hintermuller, C., Mikuljan, G., David, C., Pfeiffer, F., Stampanoni, M., and Hintermüller, C., "Advanced phase-contrast imaging using a grating interferometer," Journal of Synchrotron Radiation 16, 562-572 (2009).

[6] Schleede, S., Meinel, F., Bech, M., Herzen, J., Achterbold, K., Potdevin, G., A, M., Adam-Neumair, S., Thieme, S., Bamberg, F., Nikolaou, K., Bohla, A., Yildirim, A., Loewen, R., Gifford, M., Ruth, R., Eickelberg, O., Reiser, M., and Pfeiffer, F., "Emphysema diagnosis using X-ray dark-field imaging at a laser-driven compact synchrotron light source," Proceedings of the National Academy of Sciences of the United States of America 109, 1-6 (2012).

[7] Raupach, R. and Flohr, T., "Performance evaluation of x-ray differential phase contrast computed tomography (PCT) with respect to medical imaging," Medical Physics 39(8), 4761-4774 (2012).

[8] Thüring, T., Modregger, P., Grund, T., Kenntner, J., David, C., and Stampanoni, M., "High resolution, large field of view x-ray differential phase contrast imaging on a compact setup," Applied Physics Letters 99(4), 041111 (2011).

[9] Weitkamp, T., Diaz, A., David, C., Pfeiffer, F., Stampanoni, M., Cloetens, P., and Ziegler, E., "X-ray phase imaging with a grating interferometer," Optics Express 12(16), 6296-6304 (2005).

[10] Kenntner, J., Grund, T., Matthis, B., Boerner, M., Mohr, J., Scherer, T., Walter, M., Willner, M., Tapfer, A., Bech, M., Pfeiffer, F., Zanette, I., and Weitkamp, T., "Front- and backside structuring of gratings for phase contrast imaging with x-ray tubes," in Proceedings of SPIE, 7804, 780408 (2010).

[11] Revol, V., Kottler, C., Kaufmann, R., Jerjen, I., Lüthi, T., Cardot, F., Niedermann, P., Straumann, U., Sennhauser, U., and Urban, C., "X-ray interferometer with bent gratings: Towards larger fields of view," Nuclear Instruments and Methods in Physics Research Section A: Accelerators, Spectrometers, Detectors and Associated Equipment 648, S302-S305 (2011).

[12] Modregger, P., Pinzer, B., Thüring, T., Rutishauser, S., David, C., and Stampanoni, M., "Sensitivity of X-ray grating interferometry," Optics Express 19, 18324-18338 (2011).

[13] Thüring, T., Modregger, P., Hämmerle, S., Weiss, S., Nüesch, J., and Stampanoni, M., "Sensitivity in X-ray grating interferometry on compact systems," AIP Conference Proceedings 1466(1), 293-298 (2012).

[14] Revol, V., Kottler, C., Kaufmann, R., Straumann, U., and Urban, C., "Noise analysis of grating-based x-ray differential phase contrast imaging," Review of Scientific Instruments 81(7), 073709 (2010).

[15] Thüring, T., Modregger, P., Pinzer, B., Wang, Z., Rutishauser, S., David, C., Grund, T., Kenntner, J., and Stampanoni, M., "Towards X-ray differential phase contrast imaging on a compact setup," in Proceedings of SPIE, 2, 79611G (2011).

[16] Thüring, T., Modregger, P., Pinzer, B., Wang, Z., and Stampanoni, M., "Non-linear regularized phase retrieval for unidirectional X-ray differential phase contrast radiography," Optics Express 19(25), 2554525558 (2011).

[17] Feldkamp, L., Davis, L., and Kress, J., "Practical cone-beam algorithm," Journal of the Optical Society of America A 1(6), 612-619 (1984).

[18] Pfeiffer, F., Bunk, O., Kottler, C., and David, C., "Tomographic reconstruction of three-dimensional objects from hard X-ray differential phase contrast projection images," Nuclear Instruments and Methods in Physics Research Section A 580(2), 925-928 (2007). 